\title{
Single-shot measurement of transverse second moments using the projection method
}

\author{
Gwanghui Ha๑, ${ }^{*}$ John G. Power, Eric E. Wisniewski, Wanming Liu, and Manoel Conde \\ Argonne National Laboratory, Lemont, Illinois 60439, USA
}

(Received 3 March 2020; revised 26 October 2020; accepted 7 January 2021; published 19 January 2021)

\begin{abstract}
We introduce a diagnostic for measuring the transverse phase space (TPS) and transverse second moments of a particle beam in a single shot. The diagnostic consists of a slit followed by a simple projection beam line to project the TPS onto an imaging screen. The beam line is composed of a skew quadrupole magnet followed by a normal quadrupole magnet with an imaging screen at the end. The skew quadrupole generates a coupling between the initial $x$ and the final $y$, while the normal quadrupole removes the correlation between the initial $x$ and the initial $x^{\prime}$. Thus, the initial $\left(x, x^{\prime}\right)$ is mapped onto the final $(x, y)$. The slit is needed before the projection beam line to remove the initial $\left(y, y^{\prime}\right)$ contribution from the final $(x, y)$ image on the screen. We present simulation studies and a demonstration of the diagnostic with an experiment performed at Argonne Wakefield Accelerator facility. We also discuss the method's limitations.
\end{abstract}

DOI: 10.1103/PhysRevAccelBeams.24.012802

\section{INTRODUCTION}

The characterization of high-brightness beams is a key factor in optimizing accelerator performance in applications ranging from ion implantation to electron-based x-ray free electron lasers [1-4]. There are numerous diagnostics for measuring the individual transverse second moment of the beam (e.g., a scintillating screen for spot size or an optical transition radiation screen for divergence), but only a few methods can provide the beam's transverse phase space (TPS). This information is highly desirable, since it could be exploited to improve accelerator performance [5-8].

There are two general classes of TPS diagnostics currently in use. The first class is the pepper pot (and the related multislit mask), which has the benefit of singleshot operation but has two intrinsic limitations. First, the image captured during the measurement is composed of discrete beamlets (i.e., samples of the entire beam), and, therefore, it is only a sample of the complete TPS. Second, the image requires model-laden theory to reconstruct the TPS. The second class includes tomographic measurements which are capable of the complete measurement of the TPS but cannot do so in a single shot. Tomography is a typical example of a scanning diagnostic which is slow and provides only averaged information [9-11]. Single-shotbased diagnostics are scarce [12-14] yet in high demand.

\footnotetext{
"gha@anl.gov
}

Published by the American Physical Society under the terms of the Creative Commons Attribution 4.0 International license. Further distribution of this work must maintain attribution to the author(s) and the published article's title, journal citation, and DOI.
In this paper, we introduce a single-shot diagnostic for measuring the TPS by projecting the initial horizontal phase space $\left(x_{0}, x_{0}^{\prime}\right)$ onto a final $\left(x_{f}, y_{f}\right)$ imaging screen. For brevity, we limit our discussion to the measurement of horizontal phase space; however, it works equally well for vertical phase space. The layout of the diagnostic is a slit followed by the projection-based beam line.

The TPS diagnostic of this paper is the counterpart of the recently introduced method for measuring longitudinal phase space (LPS), wherein the LPS at the entrance of the LPS diagnostic beam line $\left(z_{0}, \delta_{0}\right)$ is projected onto the $\left(x_{f}, y_{f}\right)$ screen at the end of the LPS beam line [15-17]. In the projection-based LPS measurement, a transverse deflecting cavity (TDC) projects $z_{0} \rightarrow y_{f}$ followed by a dipole magnet that projects $\delta_{0} \rightarrow x_{f}$. However, the TDC introduces an unwanted energy gain or loss that is proportional to $x$, which shows up on the $x_{f}$ axis due to the dipole. This unwanted energy term is minimized by using a slit. In the projection-based TPS measurement, a skew quadrupole projects $x_{0} \rightarrow y_{f}$, followed by a normal quadrupole that projects the $x_{0}^{\prime} \rightarrow x_{f}$.

Similar to LPS measurement, the skew quadrupole of the TPS measurement introduces an undesired horizontal momentum change that is proportional to $y_{0}$, which shows up on the $x_{f}$ axis due to the normal quadrupole. In the special case of a flat beam, where $\varepsilon_{x} \gg \varepsilon_{y}$, the contribution of the $y$ terms to $x_{f}$ is negligible. Therefore, single-shot images of the complete TPS are obtained on the imaging screen without the use of a slit. However, similar to the situation with the LPS diagnostic, the TPS diagnostic typically needs to be implemented with a slit (either single or multiple) located before the projection beam line so it 
can be used to characterize the more general case of nonflat beams (i.e., round and elliptical beams). In this case, the use of slits means that we are not measuring the complete horizontal phase space on the final screen (similar to the LPS case). Rather, we are projecting a continuous horizontal beamlet sliced from the center of the beam along the $x$ dimension (or multiple continuous slices) onto the final screen. Note that this continuous $x$ slice is different from the pepper pot or multislit measurement which samples the beam at discrete locations along the $x$ direction.

The aim of this paper is to introduce the projection-based TPS diagnostic for measuring the TPS and the second moments in a single shot. We should note that the method is valid for beams having no correlation between $x$ and $y$. If the beam has a correlation, it would appear as a measurement error as in the case of LPS measurement and slit-scan or multislit measurements. We start with the principle of the projection beam line and discuss the limitations arising from the use of a slit, such as systematic measurement errors due to the difference between the second moments of the single slice and entire beam and the potential errors due to the final reconstruction process for multiple slices. Of course, these limitations depend on the actual condition of the incident beam. Despite these potential limitations, in the remainder of the paper, we will show that the projectionbased diagnostic fares well. It measures the second moments with high accuracy, and it produces continuous TPS images that are dense with information as opposed to the discrete images from other diagnostics such as pepper pot. This direct view of the continuous TPS provides a simple and intuitive visual tool with potential applications for machine learning. Also, the second moments $\left(\sigma_{x}, \sigma_{x}^{\prime}\right.$, $\sigma_{x x^{\prime}} / \sigma_{x}^{2}$, and $\varepsilon_{x}$ ) can easily be extracted from the TPS.

The paper is organized as follows. We begin with a description of the projection-based diagnostic by deriving analytical expressions using matrix formalism [18] in Sec. II A. We then discuss the accuracy of the projection using start-to-end numerical simulations in Sec. II B. Next, we present proof-of-principle experimental results performed at the Argonne Wakefield Accelerator (AWA) facility and benchmark its accuracy against standard TPS measurements diagnostics in Sec. II C. In Secs. III and IV, we describe the limitations that each sampling case has. Finally, in Sec. V, we conclude by considering applications of the projection-based diagnostic.

\section{PROJECTION OF TRANSVERSE PHASE SPACE}

In this section, we explain the projection beam line and its principle in the first subsection. In the following two subsections, we first explain possible error sources for the projection and its effect. Then, we show the experimental demonstration of the projection.

\section{A. Projection beam line}

We designed a beam line for the projection-based transverse diagnostic (Fig. 1) to map the initial (subscript 0) horizontal phase space at the entrance to the skew quad to a screen at the end of the beam line (subscript 2). Specifically, the beam line maps $\left(x_{0}, x_{0}^{\prime}\right)$ to $\left(y_{2}, x_{2}\right)$. The projection beam line from the entrance of the skew quad to the final screen is described in matrix formalism as

$$
\left(\begin{array}{l}
x_{2} \\
x_{2}^{\prime} \\
y_{2} \\
y_{2}^{\prime}
\end{array}\right)=\left(\begin{array}{cccc}
1 & D_{2} & 0 & 0 \\
0 & 1 & 0 & 0 \\
0 & 0 & 1 & D_{2} \\
0 & 0 & 0 & 1
\end{array}\right)\left(\begin{array}{cccc}
1 & 0 & 0 & 0 \\
-\frac{1}{f_{N Q}} & 1 & 0 & 0 \\
0 & 0 & 1 & 0 \\
0 & 0 & \frac{1}{f_{N Q}} & 1
\end{array}\right)\left(\begin{array}{cccc}
1 & D_{1} & 0 & 0 \\
0 & 1 & 0 & 0 \\
0 & 0 & 1 & D_{1} \\
0 & 0 & 0 & 1
\end{array}\right)\left(\begin{array}{cccc}
1 & 0 & 0 & 0 \\
0 & 1 & \frac{1}{f_{S Q}} & 0 \\
0 & 0 & 1 & 0 \\
\frac{1}{f_{S Q}} & 0 & 0 & 1
\end{array}\right)\left(\begin{array}{c}
x_{0} \\
x_{0}^{\prime} \\
y_{0} \\
y_{0}^{\prime}
\end{array}\right) .
$$

For clarity, we use the thin-lens approximation for the quadrupoles in this section. However, we remove this simplification for the data analysis in later sections.

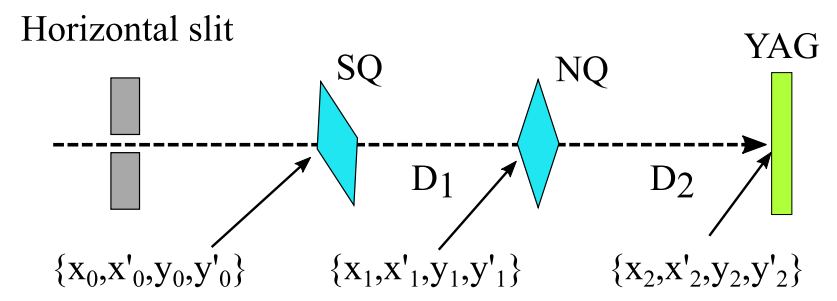

FIG. 1. Projection beam line. Schematic of the projection-based TPS diagnostic beam line for horizontal phase space measurement. The beam line consists of a horizontal slit, skew quad (SQ), normal quad (NQ), and YAG screen.
The first step is to use a skew quad of focal length $f_{S Q}$ to deliver a vertical kick that is proportional to $x$ [19] and, therefore, couples $x$ to $y^{\prime}$. The second step is to let the beam drift a length $D_{1}$, where it develops a correlation between $x_{1}$ and $x_{0}^{\prime}$ and between $y_{1}$ and $x_{0}$. The particle transport from $0 \rightarrow 1$ can be described as

$$
\begin{gathered}
x_{1}=x_{0}+D_{1} x_{0}^{\prime} \pm \frac{D_{1}}{f_{S Q}} y_{0}, \\
y_{1}= \pm \frac{D_{1}}{f_{S Q}} x_{0}+y_{0}+D_{1} y_{0}^{\prime} .
\end{gathered}
$$

At this point, we have the desired dependence of $x_{1}$ on $x_{0}^{\prime}$ and $y_{1}$ on $x_{0}$, but there are also several unwanted terms that 


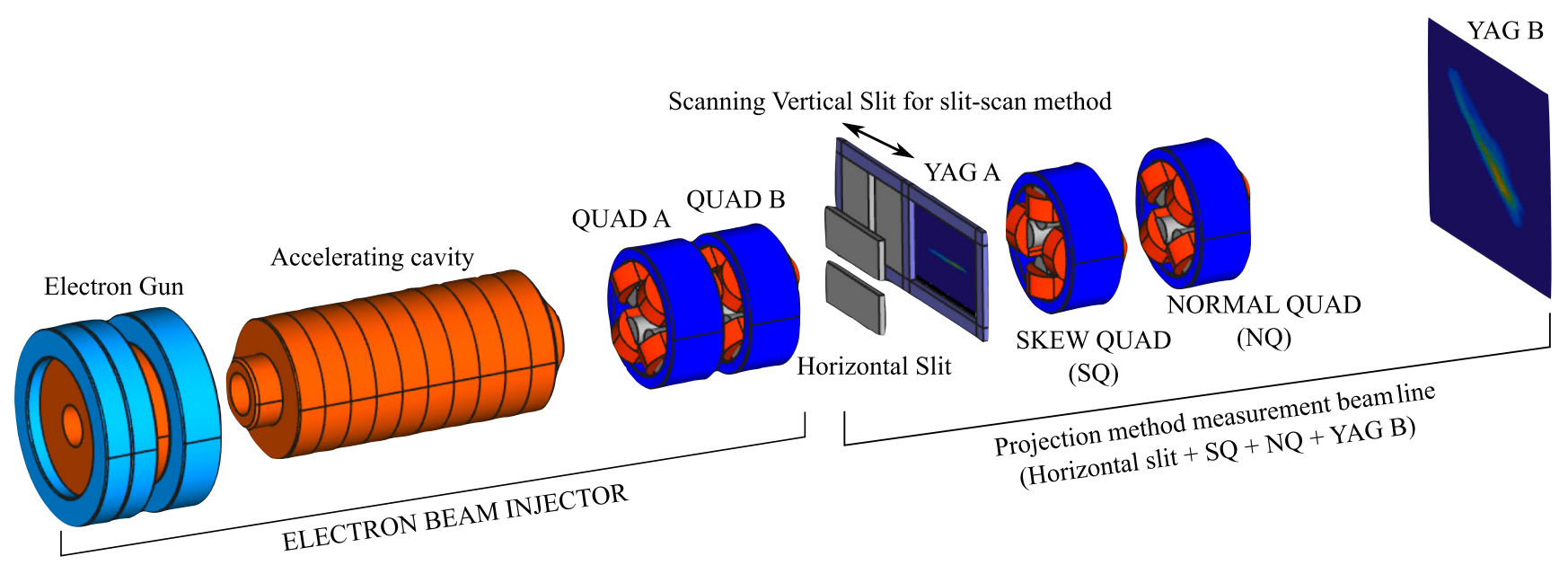

FIG. 2. Beam line configuration at the AWA facility used to demonstrate the projection-based diagnostic. The electron beam injector (electron gun to quad B) generates a variety of different beam conditions for the demonstration. The beam line for this diagnostic consists of a horizontal slit, skew and normal quads, and YAG B. The vertical slit and YAG A are not part of the projection method beam line but were added to benchmark the new method with the slit-scan method.

must be handled. Note that the polarity of the skew quadrupole does not change the result, so we use positive in the rest of the paper.

The next step is to use a normal quad of focal length $f_{N Q}$, followed by a drift of length $D_{2}$ to remove the $x_{0}$ dependence in Eq. (2). This can be done by setting $f_{N Q}=D_{2}$. Then Eqs. (2) and (3) become simpler, showing the coordinates at the location of the cerium(III)-doped yttrium-aluminum-garnet (YAG) screen:

$$
\begin{gathered}
x_{2}=D_{2} x_{0}^{\prime} \pm \frac{D_{2}}{f_{S Q}} y_{0}, \\
y_{2}= \pm \frac{2 D_{1}+D_{2}}{f_{S Q}} x_{0}+2 y_{0}+\left(2 D_{1}+D_{2}\right) y_{0}^{\prime} .
\end{gathered}
$$

At this point, we almost have the relationship we desire, but we still need to eliminate the $\left(y_{0}, y_{0}^{\prime}\right)$ terms.

The final step is to use a horizontal slit before the skew quad to minimize the impact of the $\left(y_{0}, y_{0}^{\prime}\right)$ terms in Eqs. (4) and (5). The condition required here is to make second moments of the $\left(y_{0}, y_{0}^{\prime}\right)$ terms negligible compared to $x_{0}^{\prime}$ and $x_{0}$ terms in Eqs. (4) and (5). In other words, the following two conditions should be satisfied:

$$
\begin{gathered}
\left\langle\left(D_{2} x_{0}^{\prime}\right)^{2}\right\rangle \gg\left\langle\left(\frac{D_{2}}{f_{S Q}} y_{0}\right)^{2}\right\rangle, \\
\left\langle\left(\frac{2 D_{1}+D_{2}}{f_{S Q}} x_{0}\right)^{2}\right\rangle \gg\left\langle\left[2 y_{0}+\left(2 D_{1}+D_{2}\right) y_{0}^{\prime}\right]^{2}\right\rangle .
\end{gathered}
$$

In general, two horizontal slits are necessary to satisfy the two conditions. The first horizontal slit will minimize the position $\left(y_{0}\right)$, and the second slit separated by a distance will minimize the angle $\left(y_{0}^{\prime}\right)$. However, in most cases, a single horizontal slit will minimize both terms due to the correlation between $y_{0}$ and $y_{0}^{\prime}$.
Finally, when both conditions [Eqs. (6) and (7)] are satisfied, we have

$$
\begin{gathered}
x_{2}=D_{2} x_{0}^{\prime}, \\
y_{2}= \pm \frac{2 D_{1}+D_{2}}{f_{S Q}} x_{0} .
\end{gathered}
$$

Using transfer matrix notation [18], Eqs. (8) and (9) can be written as $x_{2}=R_{12} x_{0}^{\prime}$ and $y_{2}=R_{31} x_{0}$, respectively. These equations demonstrate the principle of the projection method. They show that the projection beam line projects the $\left(x, x^{\prime}\right)$ phase space at the beginning of the beam line onto the $(x, y)$ coordinates at the location of the YAG screen when the above two conditions [Eqs. (6) and (7)] are satisfied.

Because of the slit we use to minimize vertical contributions, what we project using the beam line is the sliced beam, not the entire beam. The rest of this section uses a single horizontal slit, and we compare the second moment of a single slice and the measurement to consider feasibility and limitation of the projection only. We discuss more details of slicing and its impact on the measurement in Secs. III and IV.

\section{B. Errors in the projection}

The conditions in Eqs. (6) and (7) and the use of quadrupole magnets introduce possible error sources. We identified three factors that affect the accuracy of the projection: $\left(y_{0}, y_{0}^{\prime}\right)$ terms (as discussed in the previous section), the energy spread of the beam, and quadrupole strength errors.

We carried out numerical studies with start-to-end simulations of the beam line used in the proof-of-principle experiment (Fig. 2). The electron beam injector consists of 

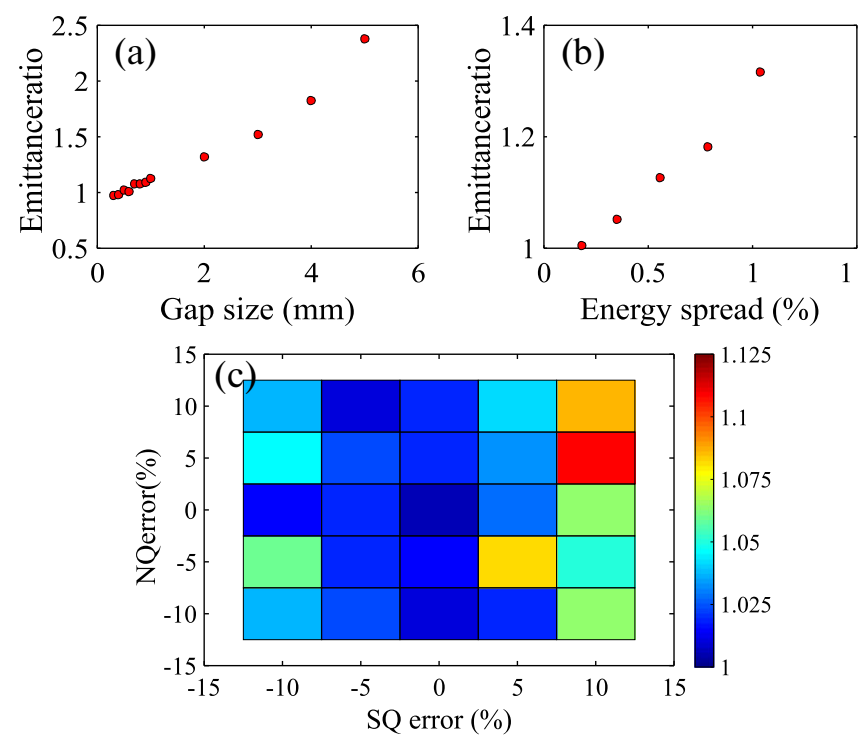

FIG. 3. Top row: ratio of measured emittance to the emittance after the horizontal slit as a function of the (a) vertical gap size and (b) energy spread. Bottom: emittance ratio for different quadrupole strength errors on the skew and the normal quadrupoles. Colors correspond to the emittance ratio. Ratios are estimated from start-to-end simulation.

a rf photocathode gun followed by an accelerating cavity and two quadrupole magnets. The gun and cavity generate a $1 \mathrm{nC}, 12.5 \mathrm{MeV}$, electron beam, while the gun solenoid and quadrupoles (quad A and quad B) control the transverse properties of the beam at the entrance to the diagnostic beam line. The diagnostic beam line begins with a horizontal slit followed by a skew quad, a normal quad, and an imaging screen at the end (YAG B). The distance between the horizontal slit and the skew quadrupole is $119.5 \mathrm{~cm}$, the skew-to-normal spacing $\left(D_{1}\right)$ is $19.8 \mathrm{~cm}$, normal quad to YAG B $\left(D_{2}\right)$ is $127.4 \mathrm{~cm}$, and quadrupole strengths $(\mathrm{k})$ are 2.3 and $2.75 \mathrm{~m}^{-1}$.

The design of the projection beam line should consider three factors that affect the accuracy of the measurements. First, an appropriately sized gap of the horizontal slit should be selected to satisfy the conditions in Eqs. (6) and (7). Second, quadrupoles introduce chromatic effects due to the energy spread of the electron beam. The chromatic effect introduces additional terms in Eqs. (8) and (9); they map particles to the wrong spot and blur the measured phase space image. Third, the actual quadrupole strengths could deviate from their theoretical setting, which will blur the image, too. These blurs on the image become errors in the second moment measurement.

In order to characterize the accuracy of the projection in the simulations, we used the ratio of the emittance measured by the projection to the actual emittance of the sliced beam. We monitor this ratio as one of the three factors is varied. Figure 3 shows the ratio of the measured emittance to the real emittance of the sliced beam.
Considering the first factor, we see that the emittance ratio increases as the gap size increases. Although only a single horizontal slit is used in the simulation, the emittance ratio is quite insensitive to its gap size. When the gap is smaller than $0.7 \mathrm{~mm}$, the emittance ratio is less than 1.1; this gap size is much larger than the typical gap size used in the slit scan $(\sim 100 \mu \mathrm{m})$.

Considering the second factor, simulations show the ratio is more sensitive to the energy spread compared to the first factor (gap). We scanned the linac phase in the simulation to control the correlated energy spread, and the corresponding emittance ratio was calculated. An energy spread of less than $0.6 \%$ provides a ratio smaller than 1.1 . This limitation is not broad enough to cover all kinds of particle beams. However, it is good enough for most electron beams, and the response to the energy spread will depend on the beam line design, too.

For the third factor, Fig. 3(c) shows the emittance ratio as colors with several different quadrupole strength errors. Less sensitivity to quadrupole strengths makes this measurement easier and more reliable. We introduced strength errors on both the skew and the normal quadrupoles. To calculate the emittance ratio, we used $R_{12}$ and $R_{31}$ from the ideal setting. The emittance ratio is quite insensitive to the quadrupole errors (up to 10\%). Compared to the normal quadrupole, the skew quadrupole strength is more sensitive to the measurement, because it is the one generating the $x$ and $y$ coupling. Note that, if one measures the transfer matrix of the given beam line and uses them for the calculation, the error from quadrupoles can be neglected.

\section{Experimental verification of projection}

The projection-based diagnostic was demonstrated at the AWA facility [20]. The details of the beam line setup are described in Sec. II B. A horizontal slit with a selectable gap (of 300 or $500 \mu \mathrm{m}$ ) is installed $92 \mathrm{~cm}$ in front of YAG A to minimize vertical contributions to the measurement. We used only a single horizontal slit in our projection beam line, because it was enough to satisfy conditions (a) and (b) mentioned in Sec. II.

The calculated elements of the transfer matrix based on the setup are given in the left column in Table I. Note that $R_{32}$ is not zero in Table I, because we now consider thicklens quadrupoles, as opposed to Eqs. (2)-(9) based on the thin-lens approximation.

The transfer matrix elements in the right column in Table I are measured by scanning two steering magnets in front of YAG A to change the incident position and angle of the beam and using YAG A and YAG B to measure the position and angle. Two-dimensional fitting provides the measured transfer matrix. Because of the nonzero elements $R_{11}$ and $R_{32}$, we used the inverse of the measured transfer matrix to recover the original TPS from the measured image at YAG B. 
TABLE I. Comparison of designed coefficients from $\left(x_{0}, x_{0}^{\prime}\right)$ to $\left(x_{2}, y_{2}\right)$ to the measurement.

\begin{tabular}{lcc}
\hline \hline Matrix element & Calculated & Measured \\
\hline$R_{11}$ & 0.00 & $0.07 \pm 0.05$ \\
$R_{12}$ & 1.32 & $1.43 \pm 0.06$ \\
$R_{31}$ & 1.04 & $0.96 \pm 0.12$ \\
$R_{32}$ & 0.33 & $0.31 \pm 0.15$ \\
\hline \hline
\end{tabular}
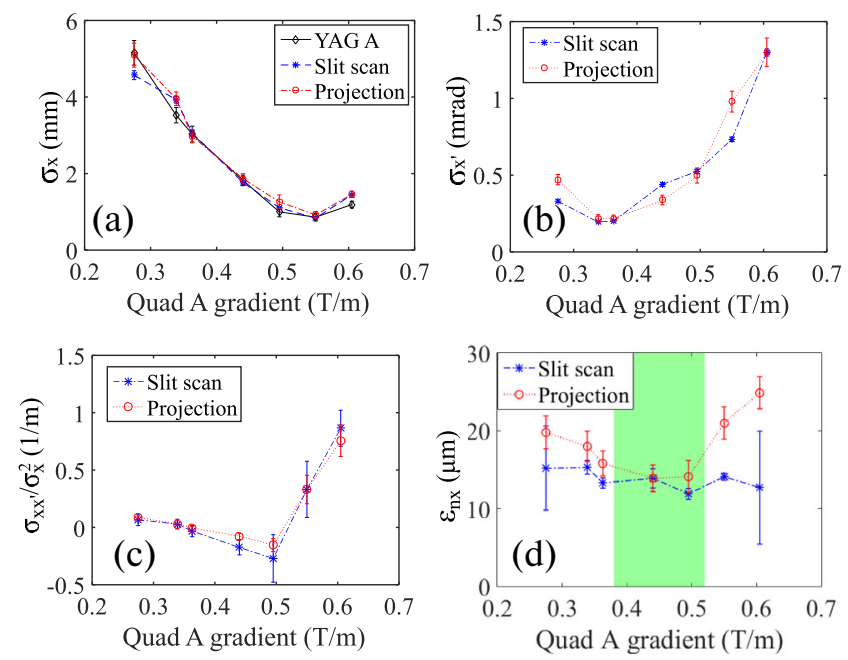

FIG. 4. Beam's transverse second moments experimentally measured by slit-scan and projection-based diagnostic. Slit scan measured the second moments of the horizontally sliced beam, and the projection beam line projects this sliced beam for the measurement. The quad B gradient is set to $-0.41 \mathrm{~T} / \mathrm{m}$, while the quad A gradient varies.
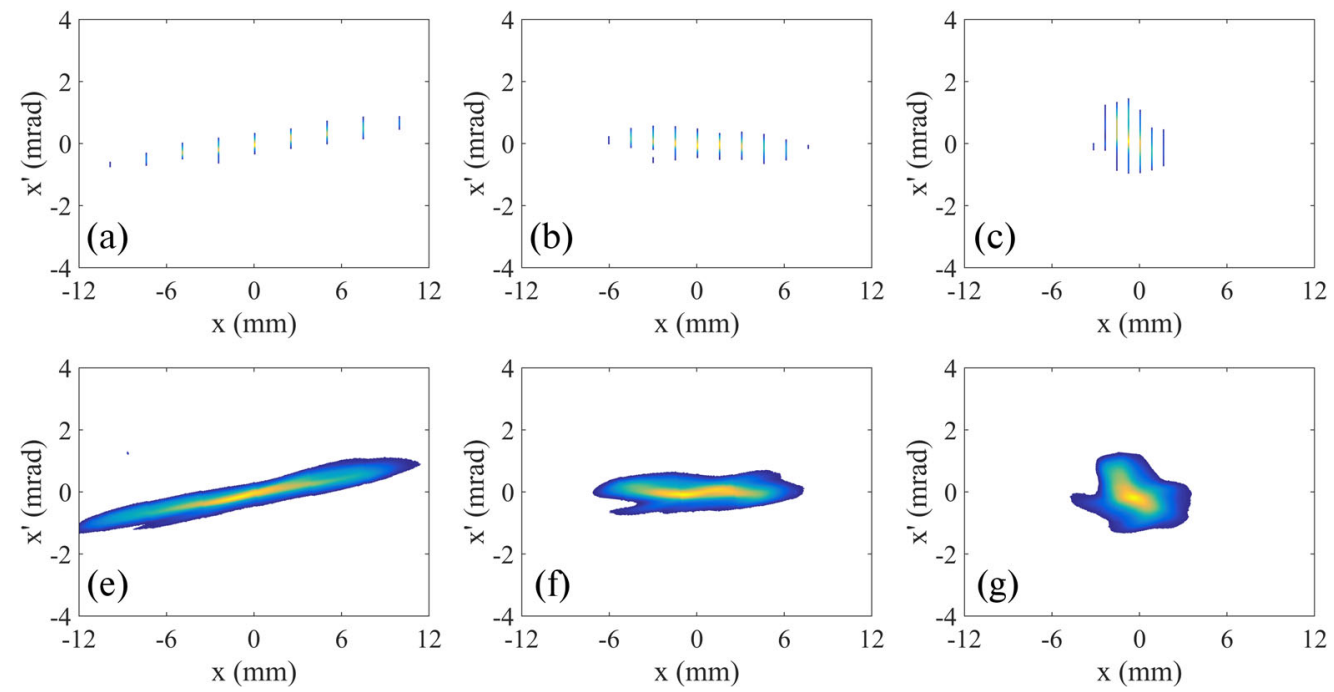

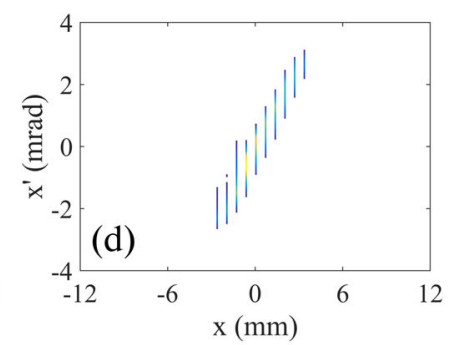

To confirm the validity of this projection, we compared its horizontal second moments $\left(\sigma_{x_{0}}, \sigma_{x_{0}^{\prime}}, \sigma_{x_{0} x_{o}^{\prime}} / \sigma_{x_{0}}^{2}\right.$, and $\left.\varepsilon_{n x}\right)$ and TPS to those of the slit scan for different quad A settings. To smooth out the beam jitter for the slit scan, 100 images were captured at YAG B for each data point. This took $500 \mathrm{~s}$ (at 2-Hz repetition) for the slit scan, because it used ten slit positions for each quad A setting. In comparison, the projection method is the single-shot method that takes $50 \mathrm{~s}$ to get 100 TPS data at the same repetition rate.

The first comparison shows that measured beam moments (a)-(c) are in good agreement, as shown in Fig. 4 ( $\sigma_{x_{0}}$ at YAG $\mathrm{A}$ is measured directly). The measured emittance (d) from the slit scan and the projection method show disagreement when the gradient of quad $\mathrm{A}$ is outside the range $0.44-0.50 \mathrm{~T} / \mathrm{m}$. The disagreement in this range is due to inaccuracy of the slit scan; it has difficulty measuring the beam halo at YAG B due to its low charge density. Note that the disagreement shows up only in the emittance, because its numerical value is generated by subtracting two large numbers.

The second comparison shows that the TPS of the beam after the horizontal slit measured with the slit scan [Figs. 5(a)-5(d)] is in good agreement with the projectionbased diagnostic [Figs. 5(e)-5(h)]. Notice that the beam halo (region of low charge density) is easily observable in Figs. 5(e)-5(h) but not in Figs. 5(a)-5(d). This explains the emittance discrepancy in Fig. 4(d).

\section{MEASUREMENT WITH SINGLE SLICING}

As described in the previous section, the projection beam line projects TPS of the beam after the slit (i.e., horizontally sliced beam), not the original TPS. Thus, it is necessary to

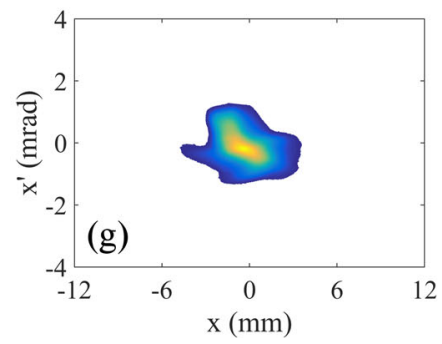

FIG. 5. Comparison of projection-based and slit-scan diagnostics. The top and bottom rows show experimentally measured TPSs using the slit-scan and projection methods, respectively. Slit scan measured the second moments of the horizontally sliced beam, and the projection beam line projects this sliced beam for the measurement. From left to right, the columns correspond to quad A gradient settings of $0.28,0.36,0.50$, and $0.61 \mathrm{~T} / \mathrm{m}$. The quad $\mathrm{B}$ gradient is $-0.41 \mathrm{~T} / \mathrm{m}$. 

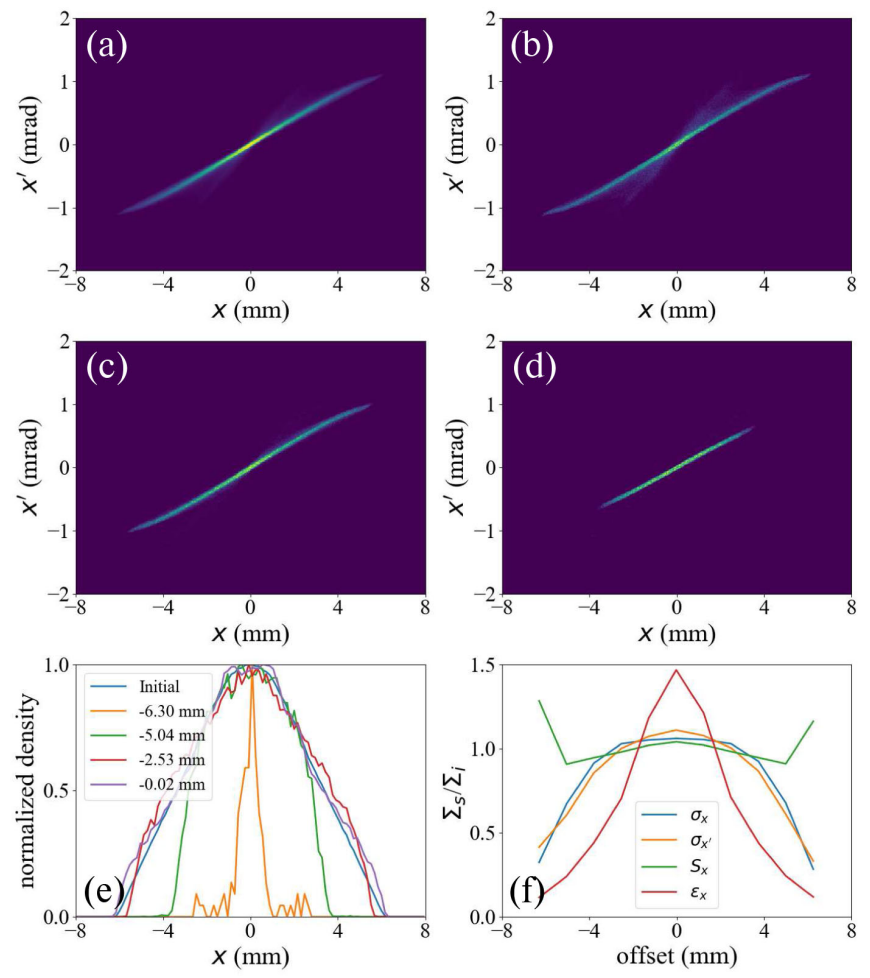

FIG. 6. (a) Horizontal phase space from start-to-end simulation. Image is taken before the horizontal slit. (b) Horizontal phase space after the slit. Here, the slit opening is located at the vertical center of the beam. (c) Horizontal phase space after the slit that is located at $y=-2.53 \mathrm{~mm}$. (d) Horizontal phase space after the slit that is located at $y=-5.04 \mathrm{~mm}$. (e) Comparison of horizontal profiles before the slit (blue) with profiles after slits located in different vertical positions. (f) Ratios of second moments before and after the slit with different vertical positions. Offset means the distance between the center of the slit and the vertical center of the beam.

understand what errors this sampling generates. In this section, we will discuss the single-slice case based on our start-to-end simulation for the beam line in Fig. 2. The next section will discuss the multislice case.

The single slice can represent the whole beam when the horizontal distribution does not have any dependency on the particle's vertical position. In other words, horizontal distributions at two arbitrary vertical positions, $y_{i}$ and $y_{j}$, should satisfy $N\left(x, y_{i}\right)=k \times N\left(x, y_{j}\right)$, where $k$ is a real number and $N$ is the particle's $x-y$ distribution. This requirement is true for ideal 2D Gaussian distribution $\left[N(x, y)=\frac{N_{0}}{2 \pi \sigma_{x} \sigma_{y}} \exp \left[-\frac{x^{2}}{2 \sigma_{x}^{2}}-\frac{y^{2}}{2 \sigma_{y}^{2}}\right]\right]$. However, this is not a realistic condition that real beams always satisfy. For example, (i) if the beam has an elliptical transverse boundary, the horizontal distribution depends on the vertical coordinate (e.g., the particle exists in $\frac{x^{2}}{\sigma_{x}}+\frac{y^{2}}{\sigma_{y}} \leq 1$ ), (ii) particle distributions can have asymmetric distribution due to incident laser distribution, nonlinear fields, time-dependent kicks, etc., and (iii) $x-y$ distribution may be tilted (i.e.,
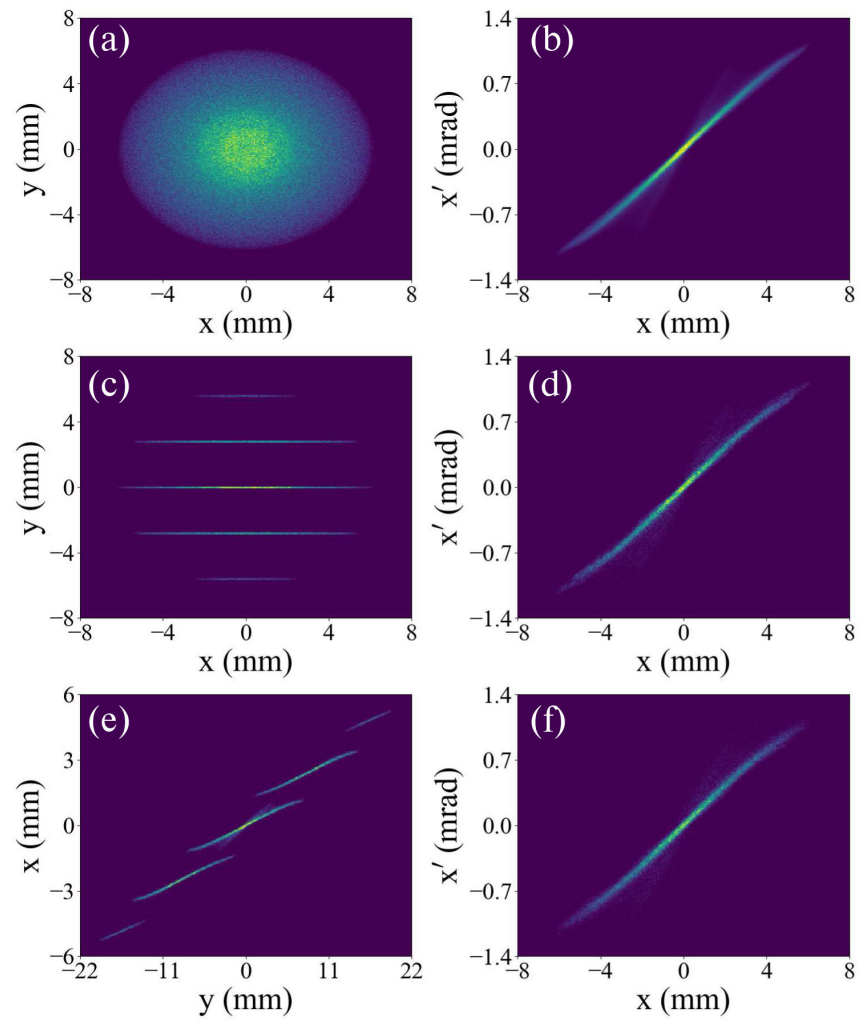

FIG. 7. Beam's $x-y$ (right) and $x-x^{\prime}$ (left) images taken at different locations are displayed. These are generated from startto-end simulation. (a) and (b) are the images before a multislit. (c) and (d) are the images after the multislit. (e) is the $x$ - $y$ beam image at the end of the beam line. (f) is the reconstructed phase space image using data from (e). Here, the reconstruction target is (b).

$\langle x y\rangle \neq 0$ ). If we measure either TPS or the second moments using a sliced distribution from the original distribution having any of these correlations, the measurement will include systematic errors.

Because it is hard to derive a simple equation for arbitrary correlations, here we limit our discussion to the start-to-end simulation case described in the previous section. Systematic errors coming from correlations should be considered during designing the projection-based diagnostics. To observe the difference between a horizontal slice and the entire beam before the slit, we compared phase space images, horizontal profiles, and second moments of different horizontal slices from different vertical positions. They are summarized in Figs. 6, and 7(a) shows the beam's original $x-y$ distribution.

The original $x-y$ distribution shows two clear $x-y$ correlations. First, the distribution has a round shape, which makes the horizontal beam size change along the vertical direction. Second, the density of the $\pm 2 \mathrm{~mm}$ region shows much higher density than the other area. This will make a difference on the horizontal profiles of slices taken at the vertical center and near the periphery of the beam.

We can observe these differences that the slicing introduces from Fig. 6. Figure 6(a) shows the TPS of the entire 
TABLE II. Comparison of calculated emittances from simulations. Second moments of the original case correspond to those of the entire beam. Second moments of the case 1 and case 2 slice correspond to the single slice and multislice's second moments, respectively. Case 1 and 2 measurement shows the estimated second moments from the reconstruction using the projection method in simulation. The multislit used for the multislice case consists of five slits with a $100 \mu \mathrm{m}$ opening, and the slits are separated by $2.8 \mathrm{~mm}$.

\begin{tabular}{lcccc}
\hline \hline Cases & $\sigma_{x}(\mathrm{~mm})$ & $\sigma_{x^{\prime}}(\mathrm{mrad})$ & $S_{x}\left(\mathrm{~m}^{-1}\right)$ & $\varepsilon_{x}(\mu \mathrm{m})$ \\
\hline Original & 2.524 & 0.528 & 0.207 & 5.120 \\
Case 1 slice & 2.667 & 0.587 & 0.216 & 7.697 \\
Case 1 measurement & 2.661 & 0.583 & 0.215 & 7.442 \\
Case 2 slice & 2.511 & 0.527 & 0.207 & 5.214 \\
Case 2 measurement & 2.523 & 0.531 & 0.208 & 5.376 \\
\hline \hline
\end{tabular}

beam before the slit, while Figs. 6(b)-6(d) show TPS of the horizontally sliced beam with different slit vertical locations. Because of the round shape of the beam, Figs. 6(b)-6(d) show different horizontal beam sizes, and corresponding horizontal profiles are clearly different, too [see Fig. 6(e)]. Figure 6(b) shows good agreement with the original one, because it covers all horizontal domains that particle exists and high density core. However, it is clear that the density ratio of the core to the periphery is much lower in the sliced image due to the round shape. These differences affect on the second moments, too. While the rms beam size, rms divergence, slope of the phase ellipse, and normalized emittance of the original beam are $2.524 \mathrm{~mm}, 0.528 \mathrm{mrad}, 0.207 \mathrm{~m}^{-1}$, and $5.120 \mu \mathrm{m}$, respectively, the slice from the center of the beam gives $2.667 \mathrm{~mm}, 0.587 \mathrm{mrad}, 0.216 \mathrm{~m}^{-1}$, and $7.697 \mu \mathrm{m}$, respectively (summarized in Table II). While other second moments have errors of $10 \%$ or less, the emittance error is $47 \%$, because the emittance is the subtraction of two numbers. It is more sensitive to small measurement errors. These errors change as the slit position changes, and it is shown in Fig. 6(f). Errors of rms beam size, rms divergence, and slope are less than $10 \%$ when the slit position is within $\pm 1.5 \sigma_{y}, \pm 1.4 \sigma_{y}$, and $\pm 2.0 \sigma_{y}$, respectively. Although the emittance error gets smaller as the slit location gets close to $\pm 1.77 \mathrm{~mm}$, this is numerical coincidence, not a good target for the measurement. These tendencies in Fig. 6(f) will depend on the original distribution, so these systematic errors should be included in the design.

Although the single slice introduces systematic errors to the measurement, measuring these second moments and TPS in a single shot can provide lots of useful information. Because these errors are systematic errors, it may be calibrated and used for the single-shot measurement. Also, these phase space and measured second moments respond to the incident beam, so it can be a powerful method to diagnose the machine status or tuning the beam line. For example, collecting all signals such as laser intensity, rf phase, etc., with these single-shot beam data can be used to diagnose jitter or drift sources of the machine and to estimate its impact on the beam. Also, we imagine that the injector tuning process can be done online while we watch the TPS in real time. Obviously, these diagnostics or tunings will be synergistic with emerging machine-learning techniques [21-23]. More details about these possible applications will be discussed in Sec. V.

\section{MEASUREMENT WITH MULTISLICING}

Because of the errors involved with using a single horizontal slice of beams having $x-y$ correlations, we next consider multislicing and reconstruct TPS using these multiple slices. Contrary to the single slicing, the multislicing should use a multislit, which is a single plate with multiple openings. Figure 7 shows the beam images from a start-to-end simulation. Each row corresponds to a different location of the beam line, before and after the horizontal slit, and the YAG screen. After the beam passes through a multislit, only a few slices remain, and it looks like Fig. 7(c). Because each slice satisfies the condition in Eqs. (6) and (7), TPS of each horizontal slice in Fig. 7(c) will be projected to the YAG screen as Fig. 7(e). Once we get TPS of multiple slices, the reconstruction requires us to move each TPS properly. Figure 7(f) shows the reconstructed TPS, and Figs. 7(b) and 7(d) show the original TPS and the one after the multislit, respectively.

Table II shows the summary of transverse second moments for different cases. As described in the previous section, the slice has different second moments than the entire beam. Especially, the emittance is changed more than $2 \mu \mathrm{m}(\sim 47 \%)$ from the original. Although the projection beam line successfully reproduces the second moments of the sliced beam, it includes a systematic error compared to the original. On the other hand, a multislit introduces a much smaller change of the second moments. Even the emittance differs by only $0.1 \mu \mathrm{m}(\sim 2 \%)$. The projection beam line reproduces these second moments after the multislit. Systematic errors in the multislicing are considerably lower than the single-slicing case.

This multislicing measurement must consider three additional factors than the single-slicing case: (i) separation of projected images at the screen, (ii) number of slits and their separation to minimize sampling errors, and (iii) moving TPS of each slice for reconstruction. To reconstruct TPS from multiple slices, we calculated weighted average positions of each slice's TPS and made them overlap by moving those TPSs. This is used to achieve TPS images in Figs. 7 and 8. This reconstruction process is a major weak point of the method, which limits the measurement. More details will be discussed in the last subsection. We discuss each factor in the rest of the section. 

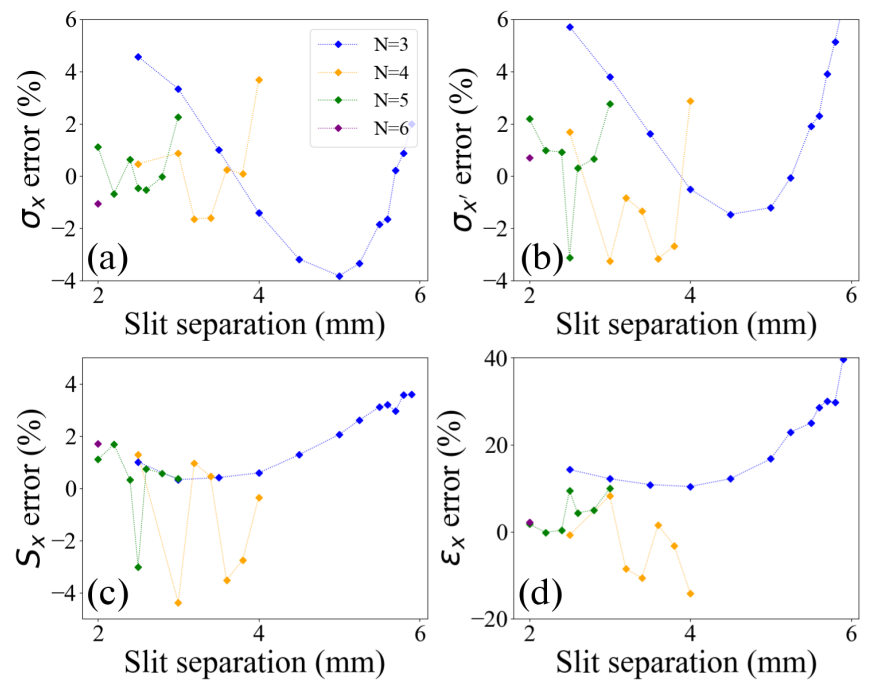

FIG. 8. Simulated measurement errors for each second moment as a function of the number of slit openings and the separation. Each panel corresponds to each second moment. Errors in figures are estimated values from the start-to-end simulation.

\section{A. Separation of projected images}

If the projected images from each horizontal slice overlap at the screen not like Fig. 7(e), it is impossible to distinguish them and obtain information. Thus, we should provide a large enough slit separation to avoid this overlap. To simplify the calculation of the overlap criteria, we assume two identical phase ellipses on YAG B whose centers are separated by $(\Delta x, \Delta y)$. Because a horizontal slice close to vertical edges has a smaller second moment than one near the core, using a larger second moment for both slices is a conservative assumption. We also assume that particles exist only within $n \sigma$ ellipses, which can be written as

$$
\frac{\sigma_{x^{\prime}}^{2} x^{2}-2 \sigma_{x x^{\prime}} x x^{\prime}+\sigma_{x}^{2} x^{\prime 2}}{\varepsilon_{x}^{2}} \leq n^{2}
$$

The orientation of ellipses will be very similar, because they are originated from the same beam. Considering two identical ellipses is valid. At the screen, the condition to separate two identical ellipses can be written as

$$
\sigma_{y_{f}}^{2} \Delta x^{2}-2 \sigma_{x_{f} y_{f}} \Delta x \Delta y+\sigma_{x_{f}}^{2} \Delta y^{2}>4 n^{2} \varepsilon_{x_{f} y_{f}}^{2} .
$$

Those coefficients $\left(\sigma_{y_{f}}^{2},-2 \sigma_{x_{f}} \sigma_{y_{f}}\right.$, and $\left.\sigma_{x_{f}}^{2}\right)$ defining an ellipse at the screen can be calculated using Eqs. (4) and (5). Then, Eq. (11) can be rewritten using beam line parameters, beam parameters after the slit, and the slit separation $(\Delta s)$ :

$$
\Delta s^{2}\left[A^{2} \sigma_{x_{0}}^{2}-2 A B \sigma_{x_{0} x_{0}^{\prime}}+B^{2} \sigma_{x_{0}^{\prime}}^{2}\right]>4 n^{2} A^{2} f_{S Q}^{2} \varepsilon_{x_{0}}^{2},
$$

where $A \equiv \frac{D_{2}\left(2 D_{1}+D_{2}\right)}{f_{S Q}^{2}}, B \equiv 2 D_{2}+D_{2}\left(2 D_{1}+D_{2}\right) S_{y_{0}}$, and $S_{y_{0}}$ is the initial vertical slope.

A large slit separation can be used to avoid the overlap of the transverse slices of the beam once the beam parameters are known. However, in some situations, the slit separation needs to be minimized, and this equation can be useful. For example, the measurement of the longitudinal slice's TPS, that we will explain in Sec. V C, requires two multislits, which generates two sets of slices. This requires minimization of the slit separation for the second multislit but maximization of the slit separation for the first multislit to keep all machine parameters in a reasonable range.

\section{B. Number of slits and separation}

Although the maximum separation of the slits guarantees the separation of the projected slices on the imaging screen, the measurement error may depend on the separation, because the slices are taken from different parts of the beam. We compared a few different cases having different numbers of slits and different slit-to-slit separations. The beam used for the study is the start-to-end simulated beam given in Fig. 7. The opening of each slit in the multislit mask was set to $100 \mu \mathrm{m}$, which is small enough size to ignore the error coming from the projection itself. Because of this small slit opening, the transmission through multislits was low. This low transmission made numerical errors on the final results.

Figure 8 shows the measurement error for each second moment for different numbers of slits $(N)$ and separations $(\Delta s)$. Note that the maximum extent of the beam in Fig. 7 has a radius of $6 \mathrm{~mm}$, so no slits are used beyond this. For $N=3$, the measurement errors show some trend rather than numerical fluctuation, but this should be the trend we can find from the single-slicing case in Fig. 6(f). The point giving $0 \%$ error is a numerical coincidence and should not be a design target. However, there is a clear difference compared to the single-slicing case. Here, rms beam size, rms divergence, and slope show reasonably small errors $(<5 \%)$ for all separations. The emittance has a much larger error than other second moments (10\%-20\%), but this is already less than half of the single-slicing case (47\%). It is also clear that moving the slit close to the periphery of the beam increases the error up to $40 \%$, which is similar to the single-slicing case.

On the other hand, $N \geq 4$ show even smaller systematic errors including the emittance case. Errors for rms beam size, rms divergence, and slope are within $2 \%$. Although errors reach to $4 \%$ for some cases, it is most likely numerical error due to the transmission issue mentioned earlier. The emittance error is mostly within $10 \%$. Clearly, more slices provide smaller errors, because we provide more information from more slices. However, the number of slit openings we can use is limited due to the separation condition. Given the maximum radial extent of the beam 
(6 $\mathrm{mm})$, the maximum number of slits we can use was $N=5$ with $\Delta s=2.8 \mathrm{~mm}$.

\section{Limitation of the method}

The projection of a multislice appears on the screen as in Fig. 7(e). Because phase spaces from each slice are separated, we should move these phase spaces to reconstruct the original phase space. However, this translation includes one unknown parameter: original vertical slope $\left(S_{y}\right)$. According to the beam transport through the projection beam line in Eqs. (4) and (5), slices vertically separated by $\Delta s$ will be shifted by $\frac{D_{2}}{f_{S Q}} \Delta s$ in the $x$ direction and $\left\{2+\left(2 D_{1}+D_{2}\right) S_{y}\right\} \Delta s$ in the $y$ direction. While other arguments are known, $S_{y}$ is an unknown parameter for this measurement. Thus, we can imagine two ways to get the reconstructed phase space.

The first way is assuming the average centers of each sliced phase space $\left(\langle x\rangle_{i}\right.$ and $\left.\left\langle x^{\prime}\right\rangle_{i}\right)$ are the same. In this case, we can calculate the weighted average of horizontal and vertical positions of each slice on the screen. Then we can move them so that their weighted centers overlap. This is the way we did it for Figs. 7 and 8. This is true for symmetric beams such as our start-to-end simulated beams, but this assumption fails when the beam has asymmetric profiles. For example, two beamlets taken from $y>0$ and $y<0$ will have different weighted centers when the beam includes a hot spot on the $x, y>0$ area. Also, if the beams is tilted on $x-y$ space, it will introduce the same problem. Although the simulation case we introduced satisfies this assumption, this method will introduce error on the measurement for beams not satisfying this assumption.

The second way is measuring averaged $S_{y}$. If we use a vertical single slit and apply opposite current direction to each quadrupole, we can measure the vertical second moments using the single slicing. As shown in Sec. III, the slope has a small error (a few percent), so it is possible to get averaged $S_{y}$ and use it as a calibration factor for the translation.

\section{APPLICATIONS OF THE PROJECTION METHOD}

There are two major advantages of the projection-based diagnostics. First, we can observe the phase space (or its scaled version) on the screen without any reconstruction process (or simple matrix multiplication). Second, horizontal slicing for the horizontal measurement helps to observe the overall shape of the phase space (see Fig. 5) rather than a discrete image from other diagnostics. In addition to the nominal purpose of the measurement, the characterization of the beam, these two advantages of the projection method may provide extra benefits on certain applications. Especially, the combination of the projection method and emerging machine-learning techniques can be synergistic [21-23].
In this section, we begin by introducing two possible synergistic applications and end by introducing a possible use of the TPS projection method for measurement of a longitudinal slice's TPS along the length of the beam.

\section{A. Online optimization}

Accelerators require a tuning process to optimize the machine settings for specific purpose. For example, injector parameters have to be optimized to achieve the minimum emittance [24-26]. Usually, machine settings start with a setting from simulation work, and they are continuously adjusted while we measure the emittance using diagnostics such as quad scan. Those methods measuring emittance provide second moments, but they do not provide the image of phase space, which includes more information that second moments do not show (e.g., nonlinearity in the phase space, etc.). Also, most of these methods are slow. Similarly, single-shot methods like pepper pot are fast and provide the phase space image, but they show only discrete sampled phase space like the top row in Fig. 5. On the other hand, the projection method provides the continuous image of the horizontal phase space (although it is the phase space of a horizontal slice) and works in a single shot.

Although we did not make the system work with machine-learning techniques, we show measured horizontal phase spaces of the beam after the injector at three different launching phases as an example. The measured data are displayed in Fig. 9. While other methods providing phase space require complicated postprocessing, the projection method requires only a matrix multiplication to the image array to achieve these images in Fig. 9. This means that operators can watch the change on the phase space and their second moments while they tune the beam line. Specifically, in this example, we can see that the slice
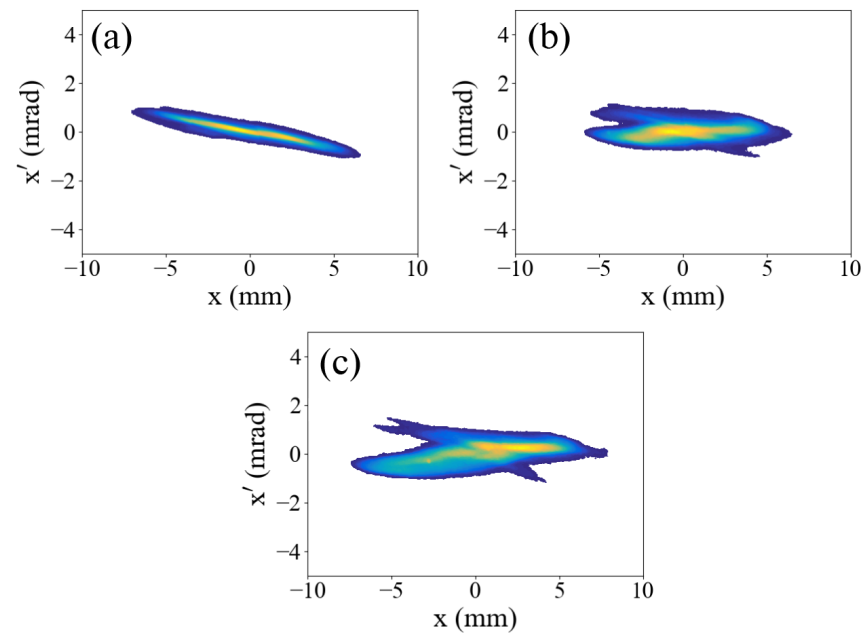

FIG. 9. Experimentally measured phase spaces with different launching phases. Single slicing is used with the projection method to measure the phase space. All machine parameters are fixed, while the launching phase is varied to $30^{\circ}, 50^{\circ}$, and $70^{\circ}$. 

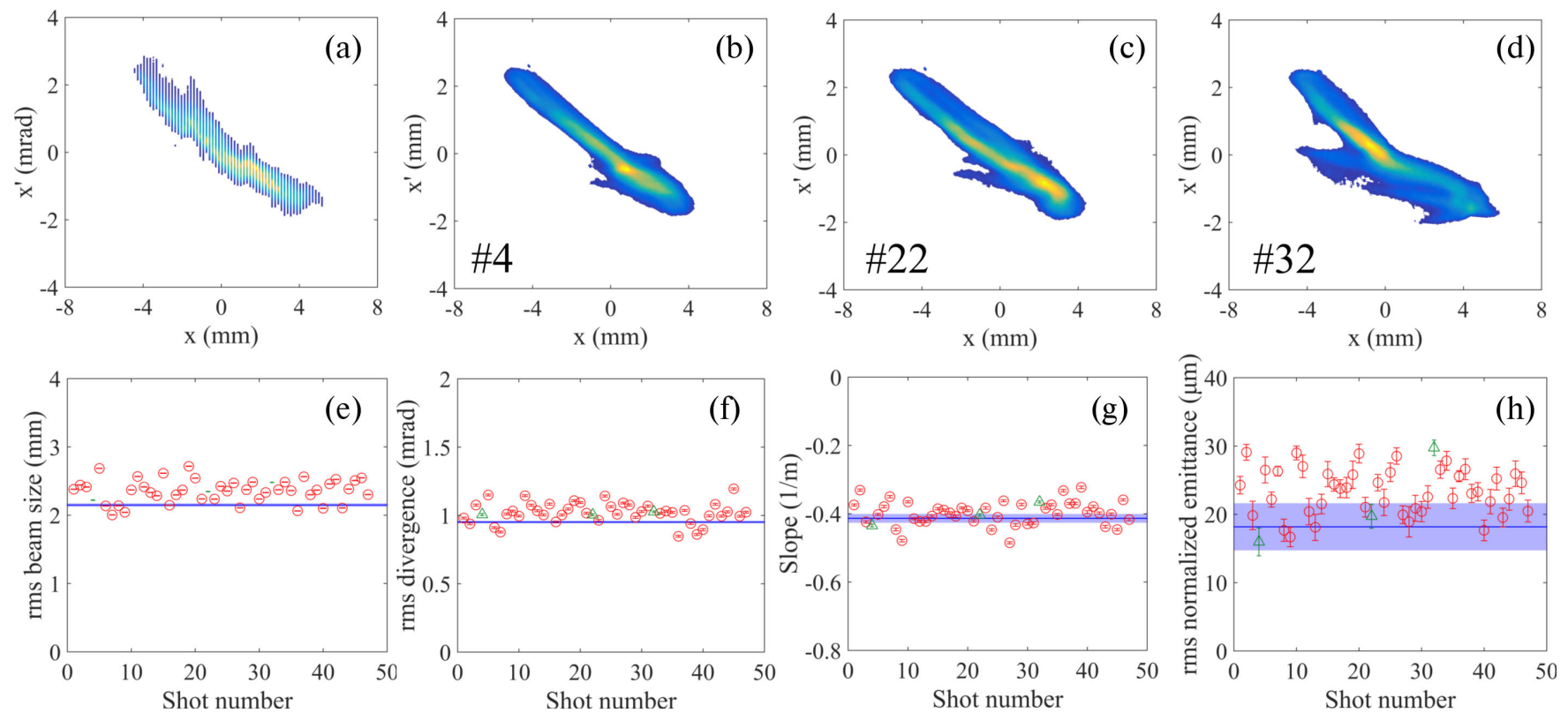

FIG. 10. Comparison of the averaged (slit-scan) and single-shot (projection-based) measurements. (a) Averaged TPS measured with the slit scan using 76 slit positions. (b)-(d) Single-shot TPS measured with the projection-based diagnostic. Each figure corresponds to the given shot numbers, whose charges are $1.63,1.62$, and $1.60 \mathrm{nC}$, respectively. (e)-(h) Horizontal beam parameters and their error estimated from the slit-scan (blue line, shade) and the projection-based diagnostic (red). Green triangles correspond to (b)-(d).

phase space from the head and tail of the bunch is reasonably aligned to the core ones at $30^{\circ}$ launching phase. On the other hand, they start to move in different ways in other launching phases. Also, there is a clear increase of the area. If other machine information and simulation data are collected together, the beam's status can be analyzed better in a shorter time than what people currently do now. This information with machine-learning algorithms would help to tune and optimize the machine fast. Also, this information may be used to develop a virtual diagnostics for online prediction [21].

\section{B. Jitter and quality degradation source detection with big data}

Similar to the previous application, we can try to find the source of beam jitter using this diagnostic. Here, we provide an experimental test case. The slit scan was used to produce Fig. 10(a), which shows the averaged TPS using a scan step size of $160 \mu \mathrm{m}$ and 76 slit locations. A total of 100 images were captured at each slit location, and the data took over $1 \mathrm{~h}$ to collect. The TPS in Fig. 10(a) is the averaged image within the charge window $(1.60 \pm 0.08 \mathrm{nC})$ and is, therefore, incapable of tracking jitter. However, the projection-based diagnostic was used to produce the single-shot images of the TPS, and three examples are shown in Figs. 10(b)-10(d). These images correspond to the fourth, 22nd, and 32nd shots with charges of $1.63,1.62$, and $1.60 \mathrm{nC}$, respectively. They were captured at the machine repetition rate and can be used to study jitter.
Beam moments measured by the two diagnostics are compared in Figs. 10(e)-10(h). The blue line shows the average beam moments, and the shaded area shows the corresponding errors measured with the slit scan. The red dots are the single-shot values of the beam moments measured with the projection-based diagnostic. The green triangles correspond to cases in Figs. 10(b)-10(d). Comparing the slit-scan to the projection-based diagnostic once again shows good agreement between the shape of the TPS [Figs. 10(a)-10(d)] and the beam moments [Figs. 10(e)$10(\mathrm{~g})$ ], except for the underestimated emittance [Fig. 10(h)] in the slit scan due to the halo (as discussed in Fig. 5).

The single-shot TPS data taken with the projection-based diagnostic provide insight into the jitter. Observe that the single-shot TPS image in Fig. 10(d) has a large halo, a boomerang shape, and a large emittance $(30 \mu \mathrm{m})$ compared with Fig. 10(b) $(18 \mu \mathrm{m})$, yet the charges of these two images differ by less than $2 \%$. This indicates that the source of emittance variation is something other than charge (e.g., laser profile jitter). To find the source of jitter, one should measure the single-shot TPS images using the projection method while also capturing key parameters such as the laser image at the virtual cathode, magnet currents, $\mathrm{rf}$ phase, and rf power. This may allow one to identify the source of the TPS jitter and eliminate it, leading to brighter and more stable beams.

\section{Single-shot measurement of the longitudinal slice's TPS}

Another attractive application is single-shot measurement of the longitudinal slice's TPS. To measure this, we 


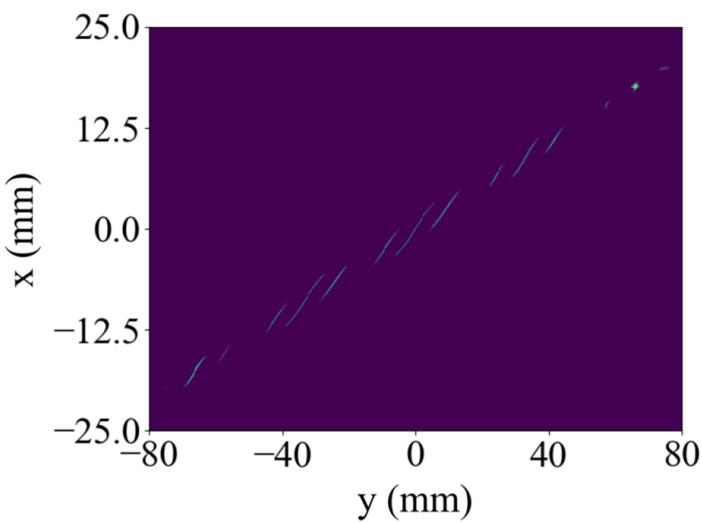

FIG. 11. $x-y$ beam image on the screen at the end of a projection beam line for a longitudinal slice measurement. The image was generated with a start-to-end simulation.

need a beam line slicing the beam in the longitudinal direction followed by a diagnostic for TPS measurement. The longitudinal slicing is usually done by a slit with a deflecting cavity or a dipole magnet, and any TPS diagnostic can follow to measure the TPS of the longitudinal slice. As mentioned in earlier sections, while other TPS diagnostics provide discrete images of the phase space, the projection method can provide a continuous image of the phase space. If multislit is used for the longitudinal slicing and the projection method is used for the TPS diagnostic, it would be possible to see multiple longitudinal slice TPSs in a single shot, and each TPS will have continuous images rather than discrete ones.

In this subsection, we present a start-to-end simulation of TPS measurement for longitudinal slices. For the longitudinal slicing, we used three quadrupoles as the focusing element, followed by a deflecting cavity and finally the longitudinal slicing multislit. We gave a large enough drift after this multislit $(3 \mathrm{~m})$ to recover reasonable beam size for the projection method. The TPS diagnostic beam line we used was the same as the one used for the other simulations in this paper.

One of the main design considerations for the longitudinal slice measurement is to avoid beamlets overlapping on the imaging screen. The total number of beamlets landing on the screen is determined by both the number of longitudinal slices and the number of transverse slices from each longitudinal slice for multislicing measurement. The final image at the screen looks like Fig. 11. There are five longitudinal slices, each of which generates three transverse slices for a total of 15 beamlets on the screen. The first multislit for the longitudinal slicing has $200 \mu \mathrm{m}$ openings with $1.8 \mathrm{~mm}$ separation and a total of five slits. The second multislit for the projection diagnostic has a total of 21 slits of width $100 \mu \mathrm{m}$ openings and is each separated by $3 \mathrm{~mm}$.

Figure 12 shows the reconstructed phase spaces for each longitudinal slice. Each longitudinal slice corresponds to
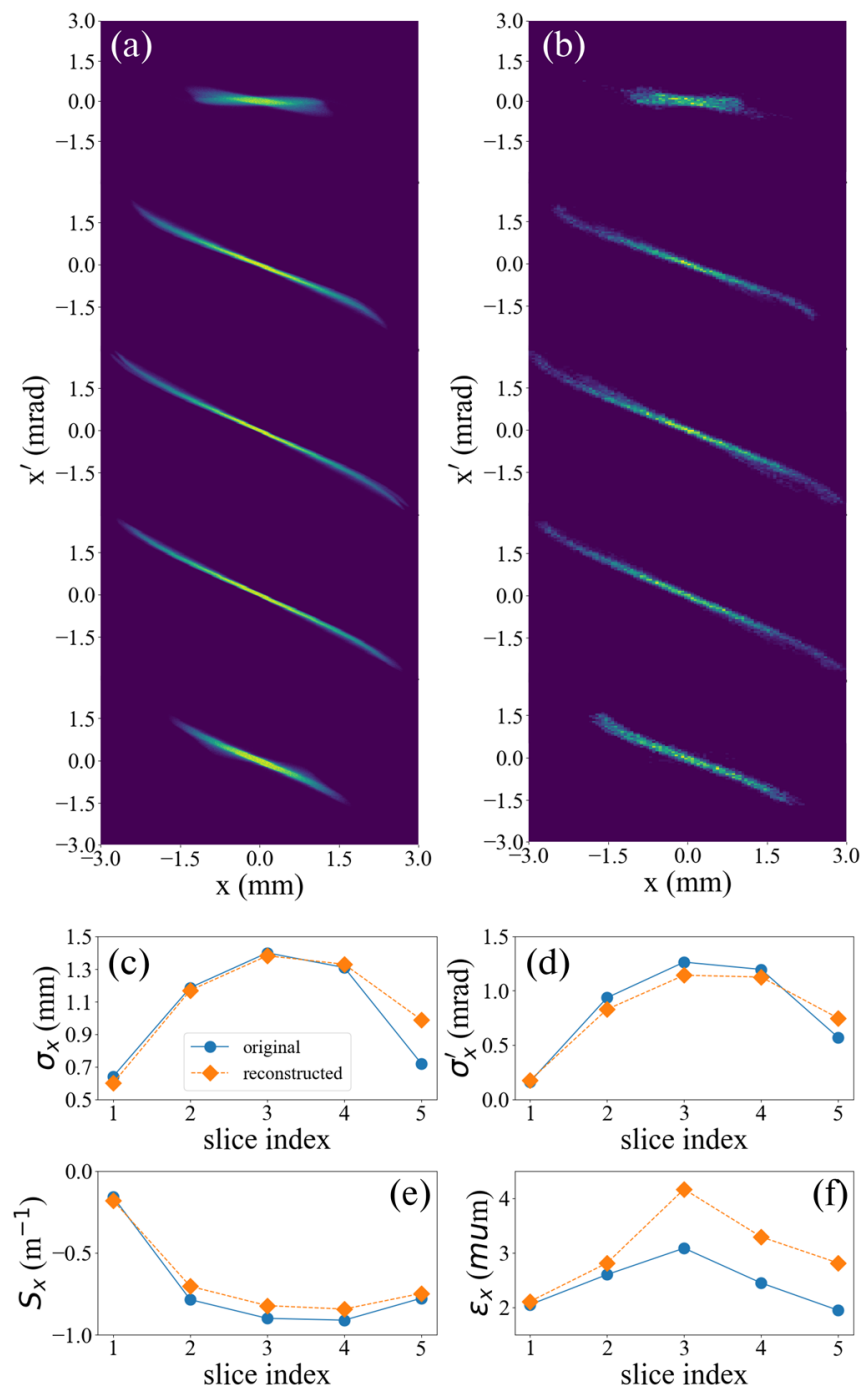

FIG. 12. Comparison of original and reconstructed slice phase spaces and second moments from start-to-end simulation. (a) $x-x^{\prime}$ phase space for different time slices. Each row corresponds to a time slice whose temporal center is $\{-4.40,-2.10,-0.20,1.75,4.00\}$ ps with a temporal width of \pm 0.6 ps. (b) Reconstructed $x$ - $x^{\prime}$ phase space using the projection method. Each row corresponds to the case in (a). (c)-(f) are a comparison of original second moments and estimated second moments from the projection method.

the temporal centers of $(-4.40,-2.10,-0.20,1.75,4.00) \mathrm{ps}$ with the width of $\pm 0.6 \mathrm{ps}$. Figure 12(a) shows the original TPS taken from the simulation immediately after the first multislit. Each longitudinal slice corresponds to a temporal center (from top to bottom). Figure 12(b) shows the corresponding reconstructed TPS using the beamlets on the final imaging screen. In Figs. 12(c)-12(f), we provided a comparison of original second moments and calculated from the reconstructed TPS. Overall, the second moments show good agreement with original values, but the emittance has a maximum $\sim 30 \%$ error. The need to keep the beamlets from overlapping is the primary cause of the emittance error. 
However, the electric field in the deflecting cavity is another source of the resolution degradation, because it increases the energy spread. If the incident beam has large enough linear chirp on the longitudinal phase space, replacing the deflecting cavity with a dipole magnet would significantly improve the resolution and relax the design tolerance. In summary, this preliminary simulation work shows that the longitudinal slice's TPS measurement is feasible, and future work should aim to minimize the required beam line space of the diagnostic and improve its resolution.

\section{CONCLUSION}

In summary, we introduced a new method, called the projection-based diagnostic, for single-shot measurement of the TPS based on the projection of $\left(x, x^{\prime}\right)$ to $(x, y)$. It uses a simple beam line consisting of a horizontal slit, a skew, and a normal quadrupole to make the projection and a fluorescent screen at the end of the beam line to capture the $(x, y)$ profile. The projection is benchmarked against the well-known slit scan; this showed that the beam moments and TPS after the slit were successfully recovered when conditions (a) and (b) were satisfied. In addition, we explored two sampling schemes works with the projection diagnostics. Single slicing can be free from the dynamics range of diagnostics, but it introduces $10 \%$ or less errors for the second moment measurements. Especially, the error for the emittance measurement was $\sim 50 \%$. On the other hand, multislicing has a dynamics range issue but provides accurate measurement on all second moments. This multislicing had an issue during the reconstruction process, so this should be considered during the design.

As the method shares the same principle as the LPS measurement using projection, this method is not applicable to beams having strong $x-y$ correlations. Thus, we recommend to consider this method when the beam has either no correlation or moderate correlation that one can apply slit-scan or multislit measurement. The correlation will appear as an error, and this error should be considered in the design. When the beam has strong $x-y$ correlation, it would be necessary to use a method such as pepper pot, which provides 4D information.

As with any method, it has limitations, but this diagnostic has many potential applications, as introduced in Sec. V. Its single-shot feature would be beneficial to beam and beam line characterization work. We introduced its possible use to online optimization and jitter or drift source detection to improve the beam quality. Also, the method can be used with a dipole magnet or a deflecting cavity to measure the beam's slice characteristics, which is another important information to understand and improve the beam quality. This single-shot measurement feature will be synergistic with recent machine-learning studies and can enhance our understanding of the accelerator system and provide a path for achieving higher brightness beams for future accelerators.

\section{ACKNOWLEDGMENTS}

This work is supported by the U.S. Department of Energy, Office of High Energy Physics, under Contract No. DE-AC02-06CH11357.

[1] M. Sarstedt, R. Thomae, H. Klein, A. Maaser, J. Müller, B. Seiler, and M. Weber, Beam profile and emittance measurements for ion implantation, Nucl. Instrum. Methods Phys. Res., Sect. B 89, 17 (1994).

[2] E. Herranz, J. L. Herraiz, P. Ibáñez, M. Pérez-Liva, R. Puebla, J. Cal-González, P. Guerra, R. Rodríguez, C. Illana, and J. M. Udías, Phase space determination from measured dose data for intraoperative electron radiation therapy, Phys. Med. Biol. 60, 375 (2015).

[3] K. Togawa, T. Shintake, T. Inagaki, K. Onoe, T. Tanaka, H. Baba, and H. Matsumoto, ceb $_{6}$ electron gun for lowemittance injector, Phys. Rev. Accel. Beams 10, 020703 (2007).

[4] R. Hajima, Emittance compensation in a return arc of an energy-recovery linac, Nucl. Instrum. Methods Phys. Res., Sect. A 528, 335 (2004).

[5] O. J. Luiten, S. B. van der Geer, M. J. de Loos, F. B. Kiewiet, and M. J. van der Wiel, How to Realize Uniform Three-Dimensional Ellipsoidal Electron Bunches, Phys. Rev. Lett. 93, 094802 (2004).

[6] C. Limborg-Deprey and P. R. Bolton, Optimum electron distributions for space charge dominated beams in photoinjectors, Nucl. Instrum. Methods Phys. Res., Sect. A 557, 106 (2006).

[7] A. Halavanau, G. Qiang, G. Ha, E. Wisniewski, P. Piot, J. G. Power, and W. Gai, Spatial control of photoemitted electron beams using a microlens-array transverse-shaping technique, Phys. Rev. Accel. Beams 20, 103404 (2017).

[8] D. Marx, J. G. Navarro, D. Cesar, J. Maxson, B. Marchetti, R. Assmann, and P. Musumeci, Single-shot reconstruction of core $4 \mathrm{~d}$ phase space of high-brightness electron beams using metal grids, Phys. Rev. Accel. Beams 21, 102802 (2018).

[9] A. V. Steenbergen, Evaluation of particle beam phase space measurement techniques, Nucl. Instrum. Methods 51, 245 (1967).

[10] C. McKee, P. O’Shea, and J. Madey, Phase space tomography of relativistic electron beams, Nucl. Instrum. Methods Phys. Res., Sect. A 358, 264 (1995).

[11] F. Ji, J. G. Navarro, P. Musumeci, D. B. Durham, A. M. Minor, and D. Filippetto, Knife-edge based measurement of the $4 \mathrm{~d}$ transverse phase space of electron beams with picometer-scale emittance, Phys. Rev. Accel. Beams 22, 082801 (2019).

[12] A. Septier, Applied Charged Particle Optics (Academic, New York, 1980).

[13] C. A. Lindstrøm, R. D’Arcy, M. J. Garland, P. Gonzalez, B. Schmidt, S. Schröder, S. Wesch, and J. Osterhoff, Matching small beta functions using centroid jitter and two beam position monitors, arXiv:2002.06022.

[14] R. K. Li, K. G. Roberts, C. M. Scoby, H. To, and P. Musumeci, Nanometer emittance ultralow charge beams 
from rf photoinjectors, Phys. Rev. Accel. Beams 15, 090702 (2012).

[15] J. T. Moody, P. Musumeci, M. S. Gutierrez, J. B. Rosenzweig, and C. M. Scoby, Longitudinal phase space characterization of the blow-out regime of $\mathrm{rf}$ photoinjector operation, Phys. Rev. Accel. Beams 12, 070704 (2009).

[16] M. Rihaoui, D. Mihalcea, W. Gai, P. Piot, and J. G. Power, Design and analysis of a single-shot longitudinal phase space measurement diagnostics at the Argonne Wakefield Accelerator, AIP Conf. Proc. 1299, 570 (2010).

[17] Q. Gao, J. Shi, H. Chen, G. Ha, J. G. Power, M. Conde, and W. Gai, Single-shot wakefield measurement system, Phys. Rev. Accel. Beams 21, 062801 (2018).

[18] A. W. Chao, K. H. Mess, M. Tigner, and F. Zimmermann, Handbook of Accelerator Physics and Engineering (World Scientific, Singapore, 2013).

[19] M. Conte and W. W. MacKay, An Introduction to the Physics of Particle Accelerators (World Scientific, Singapore, 2008).

[20] M. Conde, D. S. Doran, W. Gai, W. Liu, J. G. Power, J. Shao, C. Whiteford, E. Wisniewski, S. Antipov, C. Jing, J. Qiu, N. Neveu, Y. Wang, G. Ha, Q. Gao, and L. Zheng, Research program and recent results at the Argonne Wakefield Accelerator Facility (AWA), in Proceedings of IPAC17, Copenhagen, Denmark (JACoW, Geneva, Switzerland, 2017), pp. 2885-2887.
[21] C. Emma, A. Edelen, M. J. Hogan, B. O’Shea, G. White, and V. Yakimenko, Machine learning-based longitudinal phase space prediction of particle accelerators, Phys. Rev. Accel. Beams 21, 112802 (2018).

[22] A. Scheinker, D. Bohler, S. Tomin, R. Kammering, I. Zagorodnov, H. Schlarb, M. Scholz, B. Beutner, and W. Decking, Model-independent tuning for maximizing free electron laser pulse energy, Phys. Rev. Accel. Beams 22, 082802 (2019).

[23] A. Edelen, N. Neveu, M. Frey, Y. Huber, C. Mayes, and A. Adelmann, Machine learning for orders of magnitude speedup in multiobjective optimization of particle accelerator systems, Phys. Rev. Accel. Beams 23, 044601 (2020).

[24] L. Serafini and J. B. Rosenzweig, Envelope analysis of intense relativistic quasilaminar beams in rf photoinjectors: A theory of emittance compensation, Phys. Rev. E 55, 7565 (1997).

[25] D. H. Dowell, F. Zhou, and J. Schmerge, Exact cancellation of emittance growth due to coupled transverse dynamics in solenoids and rf couplers, Phys. Rev. Accel. Beams 21, 010101 (2018).

[26] L. Zheng, J. Shao, Y. Du, J. G. Power, E. E. Wisniewski, W. Liu, C. E. Whiteford, M. Conde, S. Doran, C. Jing, C. Tang, and W. Gai, Experimental demonstration of the correction of coupled-transverse-dynamics aberration in an rf photoinjector, Phys. Rev. Accel. Beams 22, 072805 (2019). 